EPJ Web of Conferences 35, 05001 (2012)

DOI: $10.1051 /$ epjconf/20123505001

(C) Owned by the authors, published by EDP Sciences, 2012

\title{
Dynamical approach to fusion-fission process in superheavy mass region
}

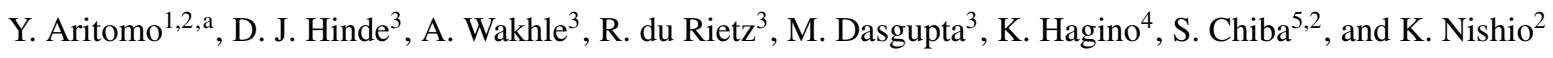 \\ 1 Flerov Laboratory of Nuclear Reactions, JINR, Dubna, 141980, Russia \\ 2 Japan Atomic Energy Agency, Tokai, Ibaraki, 319-1195, Japan \\ 3 Department of Nuclear Physics, Research School of Physics and Engineering, The Australian National University, ACT \\ 0200, Australia \\ 4 Department of Physics, Tohoku University, Sendai 980-8578, Japan \\ 5 Research Laboratory for Nuclear Reactors, Tokyo Institute of Technology, Tokyo 152-8550, Japan
}

\begin{abstract}
In order to describe heavy-ion fusion reactions around the Coulomb barrier with an actinide target nucleus, we propose a model which combines the coupled-channels approach and a fluctuation-dissipation model for dynamical calculations. This model takes into account couplings to the collective states of the interacting nuclei in the penetration of the Coulomb barrier and the subsequent dynamical evolution of a nuclear shape from the contact configuration. In the fluctuation-dissipation model with a Langevin equation, the effect of nuclear orientation at the initial impact on the prolately deformed target nucleus is considered. Fusion-fission, quasifission and deep quasi-fission are separated as different Langevin trajectories on the potential energy surface. Using this model, we analyze the experimental data for the mass distribution of fission fragments (MDFF) in the reaction of ${ }^{36} \mathrm{~S}+{ }^{238} \mathrm{U}$ at several incident energies around the Coulomb barrier.
\end{abstract}

\section{Introduction}

The prediction of the existence of the "Island of Stability" in the nuclear chart has encouraged searches of new elements. The synthesis of these superheavy elements has been carried out using heavy-ion fusion reactions between stable nuclei. An important quantity for a prediction of the cross section is the probability of fusion after the interacting nuclei have the initial contact. Due to the complexity of the process, however, a good method for predicting the fusion probability has not been well established. Furthermore, actinide nuclei are prolately deformed and the effect of nuclear orientation on fusion probability has been established [1,2]. The collision on the polar side has a large probability to disintegrate, whereas the collision on the equatorial side has a larger fusion probability. Below the barrier, the effect of nuclear orientation has been well established in the approaching phase of the reaction using the coupled-channels (CC) approach [3-5]. So nuclear orientation needs to be introduced as an additional degree of freedom.

However, it is still difficult to calculate the adiabatic potential energy to be used in the model with the twocenter parametrization for subsequent shapes of the nuclear system, starting from the configuration of arbitrarily oriented two deformed nuclei touching each other to the spherical compound nuclei. In a previous paper [6], to estimate the capture and fusion cross sections, we only considered the spherical-spherical configuration as the first approximation, limiting to the energy region above the Bass barrier.

In this paper, we propose a new model which can avoid this difficulty. Our strategy to calculate the fusion proba-

\footnotetext{
a e-mail: aritomo24@muj.biglobe.ne.jp
}

bility is to use the unified model [7], which has been developed by the FLNR theory group. The model can describe every entrance and exit channel in heavy-ion collisions, and can calculate also the time evolution of the nuclear shape, where the binary decay of the composite system (fission) can be treated. A trajectory calculation is performed on the time-dependent unified potential energy surface by using the Langevin equation. In the trajectory analysis, different types of fission processes can be separated: fusion-fission (FF) and quasi-fission $(\mathrm{QF})$. We define the compound nucleus region (that is, the fusion box) by referring to the ridge of the fission barrier in the coordinate space [8]. Fusion-fission is fission following the formation of a compound nucleus; therefore, the FF label is applied to cases where the trajectory enters the compound nucleus region before undergoing fission. The trajectories which enter in the fusion box are distinguished as the FF process. Quasi-fission, on the other hand, is the re-separation process which does not form a compoundnucleus. In our model, it corresponds to trajectories that do not enter into the fusion box and go to the re-separation region. The model should be constrained or checked by experiments by investigating whether the calculation can reproduce the measured fission spectra such as mass and total kinetic energy distributions.

Recently, the mass distributions of fission fragments (MDFF) for the reactions ${ }^{36,34} \mathrm{~S}+{ }^{238} \mathrm{U}$ and ${ }^{30} \mathrm{Si}+{ }^{238} \mathrm{U}$ at several incident energies around the Coulomb barrier were measured by the Japan Atomic Energy Agency (JAEA) [9]. One of the findings in the experiment is that the mass asymmetry in quasi-fission is different between the ${ }^{30} \mathrm{Si}+{ }^{238} \mathrm{U}$ and ${ }^{36,34} \mathrm{~S}+{ }^{238} \mathrm{U}$ systems at low incident energies. In this work, we attempt to analyze these data in order to understand the reaction mechanisms. To describe heavyion fusion reactions around and below the Coulomb bar- 
rier with actinide target nuclei, we propose a model which combines the CC approach [10] and the Langevin calculation [8].

The paper is organized as follows. In Sec. 2, we detail the framework of the new model, which combines the CC method and the dynamical Langevin calculation. In Sec. 3, we show the results for the cross sections and MDFF at several incident energies for the reaction of ${ }^{36} \mathrm{~S}+{ }^{236} \mathrm{U}$. In Sec. 4 , we present a summary of this study and further discussion.

\section{Model}

The penetration probability for the $\ell$-th partial wave, $T_{\ell}$, is obtained by the CC approach. The capture cross sections are given by

$$
\sigma_{\text {cap }}\left(E_{\mathrm{cm}} ; \theta\right)=\frac{\pi}{k^{2}} \sum_{\ell=0}^{\infty}(2 \ell+1) T_{\ell}\left(E_{\mathrm{cm}} ; \theta\right)
$$

where $k$ is the wave number of the incident flux.

The fusion cross section is calculated by multiplying the probability to form a compound nucleus, $P_{\mathrm{CN}}$ with the capture probability, $T_{\ell}\left(E_{c m} ; \theta\right)$, at each incident angle $\theta$ and integrating it over the solid angle as

$$
\sigma_{\text {fus }}\left(E_{\mathrm{cm}}\right)=\int_{0}^{1} d(\cos \theta) \sigma_{\text {fus }}\left(E_{\mathrm{cm}} ; \theta\right),
$$

with

$$
\sigma_{\text {fus }}\left(E_{\mathrm{cm}} ; \theta\right)=\frac{\pi}{k^{2}} \sum_{\ell=0}^{\infty}(2 \ell+1) T_{\ell}\left(E_{\mathrm{cm}} ; \theta\right) P_{\mathrm{CN}}\left(E_{\mathrm{cm}}, \ell, \theta\right) .
$$

The formation probability $P_{\mathrm{CN}}$ is estimated with the dynamical calculation using the Langevin equation.

After the projectile enters with an arbitrary orientation relative to the symmetry axis of the deformed target nucleus, the collision is replaced by the one from the polarside of the target nucleus and the trajectory calculation starts from the configuration corresponding to the touching distance $z_{\text {touch }}$. That is, we consider only the nose-tonose configuration. The detail is explained in reference [11]. In this stage, we assume that the potential has been shifted to the adiabatic potential from the diabatic one. In the reactions of ${ }^{238} \mathrm{U}$, the static deformation of $\beta_{2}=0.215$ $(\delta \sim 0.2)$ is used.

The nuclear shape is defined by the two-center parametrization, which has three deformation parameters, $z_{0}, \delta$, and $\alpha . z_{0}$ is the distance between two potential centers, while $\alpha=\left(A_{1}-A_{2}\right) /\left(A_{1}+A_{2}\right)$ is the mass asymmetry of the colliding nuclei, where $A_{1}$ and $A_{2}$ denote the mass numbers of heavy and light nuclei, respectively. $\delta$ denotes the deformation of the fragments, and is defined by $\delta=3(a-b) /(2 a+b)$, where $a$ and $b$ are the half length of the axes of an ellipse in the $z_{0}$ and $\rho$ directions of the cylindrical coordinate, respectively. We assume that each fragment has the same deformation. The nuclear shape and the shape parameters are described in Fig. 1.

Since we employ the CC model for the approaching phase to describe the penetration of the Coulomb barrier, the two-body part of the unified mode is omitted [6,7]

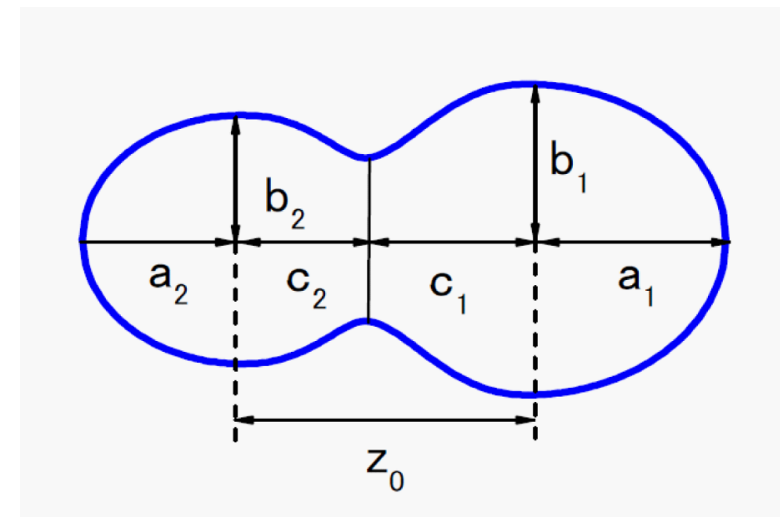

Fig. 1. Nuclear shape with the two-center parametrization.

in our calculations. The multidimensional Langevin equations [6] are thus simplified as

$$
\begin{aligned}
\frac{d q_{i}}{d t} & =\left(m^{-1}\right)_{i j} p_{j} \\
\frac{d p_{i}}{d t}=-\frac{\partial V}{\partial q_{i}} & -\frac{1}{2} \frac{\partial}{\partial q_{i}}\left(m^{-1}\right)_{j k} p_{j} p_{k} \\
& \quad-\gamma_{i j}\left(m^{-1}\right)_{j k} p_{k}+g_{i j} R_{j}(t),
\end{aligned}
$$

where $p_{i}=d q_{i} / d t$ with $i=\{z, \delta, \alpha\}$. The summation is performed over repeated indices. In the Langevin equation, $m_{i j}$ and $\gamma_{i j}$ are the shape-dependent collective inertia parameter and the friction tensor, respectively. The wall-andwindow one-body dissipation is adopted for the friction tensor. A hydrodynamical inertia tensor is adopted with the Werner-Wheeler approximation for the velocity field. The normalized random force $R_{i}(t)$ is assumed to be white noise, i.e., $\left\langle R_{i}(t)\right\rangle=0$ and $\left\langle R_{i}\left(t_{1}\right) R_{j}\left(t_{2}\right)\right\rangle=2 \delta_{i j} \delta\left(t_{1}-t_{2}\right)$. The strength of the random force $g_{i j}$ is given by $\gamma_{i j} T=$ $\sum_{k} g_{i j} g_{j k}$.

The fusion probability $P_{\mathrm{CN}}$ is determined in our model calculation by identifying the different trajectories on the deformation space. It is equivalent to the number of trajectories of compound-nucleus fission normalized to all the fission events. Formation of the compound nucleus is defined as the case in which a trajectory enters in the fusion box in the adiabatic potential energy surface. $P_{\mathrm{CN}}$ is described by using the total number of events $N$ and the number of FF trajectories $N_{\mathrm{FF}}$,

$$
P_{\mathrm{CN}}=\frac{N_{\mathrm{FF}}}{N} .
$$

\section{Mass distribution of fission fragments and cross sections}

Recently MDFF and the fission cross sections ( $\left.\sigma_{\text {fiss }}\right)$ for the the ${ }^{36,34} \mathrm{~S}+{ }^{238} \mathrm{U}$ reactions were measured by the JAEA group [9]. In the experiment, fission events were selected in which the momentum of the projectile is fully transferred to the composite system. For these systems, the fission cross sections are almost equal to those of the projectiles captured inside the Coulomb barrier, $\sigma_{\text {cap. }}$.

We first analyze these systems with the new model proposed in the previous section. The dashed-dot curve in Fig. 


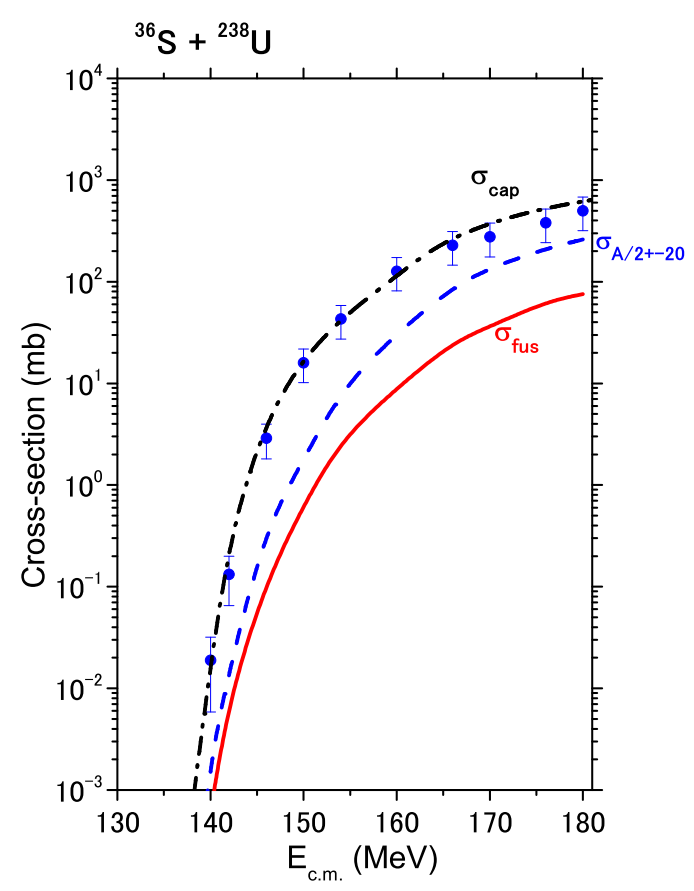

Fig. 2. (Colour online) Excitation functions of $\sigma_{\text {cap }}, \sigma_{A / 2 \pm 20}$ and $\sigma_{\text {fus }}$ for the ${ }^{36} \mathrm{~S}+{ }^{238} \mathrm{U}$ reaction. The experimental data of $\sigma_{\text {fiss }}$, denoted by the circles, are taken from [9].

2 shows the calculated capture cross sections based on the $\mathrm{CC}$ model for the the reaction ${ }^{36} \mathrm{~S}+{ }^{238} \mathrm{U}$ as a function of the incident energy. The experimental data are taken from [9]. As in [9], we use the computer code CCDEGEN [10] by taking into account the static deformation for ${ }^{238} \mathrm{U}$ with $\left(\beta_{2}, \beta_{4}\right)=(0.275,0.05)$. One can see that this calculation reproduces the measured cross sections down to the lowest incident energy below the Bass barrier $\left(V_{\text {Bass }}=158.8\right.$ $\mathrm{MeV}$ ) [12].

The solid curve in figure 2 shows the fusion cross section $\sigma_{\text {fus }}$ obtained by the new model with the CC and the Langevin equation. The dashed line shown in figure 2 denotes the cross section $\sigma_{A / 2 \pm 20}$, which is derived from the yield of the fission fragments whose mass number is located within \pm 20 around the symmetric fission $A_{\mathrm{CN}} / 2$. Notice that the fusion cross sections $\sigma_{\text {fus }}$ are significantly value is one or half order of magnitude smaller than $\sigma_{A / 2 \pm 20}$. This indicates that the mass symmetric fission does not necessarily originate from the compoundnucleus state.

With the new approach, by considering the nuclear shapes at the contact configuration for each orientation, we can also obtain the cross sections below the Bass barrier region.

The results for the MDFF for the reaction of ${ }^{36} \mathrm{~S}+{ }^{238} \mathrm{U}$ are compared with the experimental data [9] in figure 3 at seven incident energies from $E_{\mathrm{cm}}=148.0\left(E^{*}=31.5\right) \mathrm{MeV}$ to $E_{\mathrm{cm}}=176.0\left(E^{*}=61.5\right) \mathrm{MeV}$. At high incident energies, the mass distribution has a Gaussian-like shape centered at the symmetric mass division, whereas the massasymmetric fission fragments dominate at low incident energies. The mass-asymmetric fission produces nuclei in the vicinity of the doubly-closed shell nuclei, ${ }^{208} \mathrm{~Pb}$ and ${ }^{78} \mathrm{Ni}$.

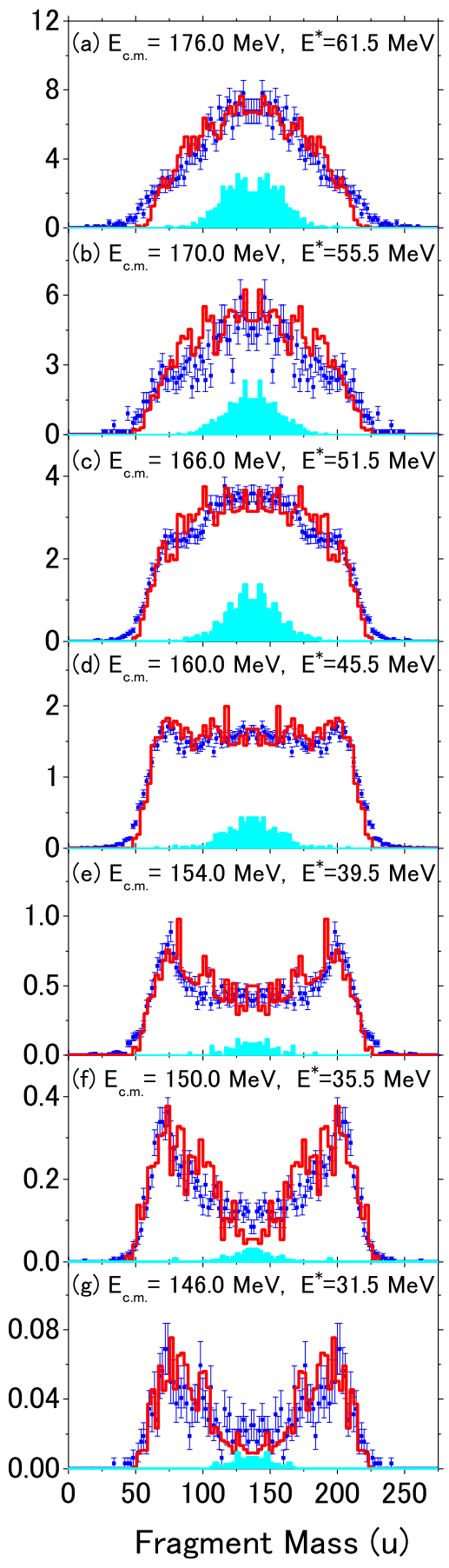

Fig. 3. (Colour online) Mass distributions of fission fragments for the reaction of ${ }^{36} \mathrm{~S}+{ }^{238} \mathrm{U}$. The experimental data and the calculated results are denoted by the circles [9] and the histograms, respectively. The shaded areas show the calculated fusion-fission events. 
The trend of the experimental data, i.e., the incident energy dependence of MDFF, is well reproduced by the calculation. The mass-asymmetry with $A_{\mathrm{H}}=200$ at sub-barrier energies is also well reproduced.

In figure 3 we also plot the fusion-fission events as filled histograms. Apparently, the compound-nucleus fission has a mass-symmetric shape, and the observed massasymmetric fission that dominates at the low incident energies is classified as QF. The strong energy dependence of the MDFF can be understood in terms of the orientation effect on the fusion and QF. The collisions on the polar side have a large probability to disintegrate as QF, whereas the collisions on the equatorial side have a larger fusion probability. The calculation also suggests that the measured mass-symmetric fission fragment has an origin other than the compound nucleus fission. Such an event is defined as a deep quasi-fission process (DQF).

\section{Summary and Outlook}

We developed a new dynamical model to describe heavyion induced fission, in which the effects of static nuclear deformation of a target nucleus are taken into account by considering all the orientation angles of the symmetry axis of the target nucleus. The orientation effects are included both in the barrier penetration process and in the evolution of the nuclear shape. The former process is described with the CC model. After the nuclear contact point, we switch to the dynamical calculation starting at the touching point assuming a nose-to-nose configuration. With this model, the calculation could be extended to energies below the Coulomb barrier for the first time.

By analyzing the Langevin trajectories, we could distinguish three different fission processes, QF, DQF and FF. The mass-asymmetric fission in ${ }^{36} \mathrm{~S}+{ }^{238} \mathrm{U}$ at $A_{L} / A_{H}=$ $74 / 200$ observed at low incident energies are from QF. We discussed the reaction ${ }^{30} \mathrm{Si}+{ }^{238} \mathrm{U}$ in the reference [11]. The mass-asymmetric fission with $A_{L} / A_{H}=90 / 178$ in ${ }^{30} \mathrm{Si}+{ }^{238} \mathrm{U}$ observed at sub-barrier energy is also from $\mathrm{QF}$, whereas the peak at the symmetric fission of $A=135$ indicates that the fission occurs with some time delay and originates from FF and DQF.

In this model, one can determine the fusion probability by selecting trajectories which enter the fusion box. The reproduction of the experimental MDFF in this model can be the ground to support the calculated fusion probability. Furthermore, the generalized formula proposed in this model has a potential to simulate any kind of heavyion induced reactions in the approaching phase, such as a nucleon-transfer reaction, and to predict cross sections for the production of new nuclei.

Recently, the mass-angle distributions of fission fragments using targets of W [13], Th and U [14] are measured by the group of the Australian National University. These data give the reaction time scale and reaction mechanism. Using the model, we are analyzing the data and clarifying the reaction mechanism.

\section{Acknowledgments}

The numerical calculations were carried out on SR16000 at YITP at Kyoto University. This work was supported by the
Japanese Ministry of Education, Culture, Sports, Science and Technology by Grant-in-Aid for Scientific Research under the program number (C) 22540262.

\section{References}

1. S. Mitsuoka, H. Ikezoe, K. Nishio, and J. Lu, Phys. Rev. C 62, 054603 (2000).

2. K. Nishio, H. Ikezoe, S. Mitsuoka, and J. Lu, Phys. Rev. C 62, 014602 (2000) ; K. Nishio, H. Ikezoe, S. Mitsuoka, K. Satou, and J. Lu, Phys. Rev. C 63, 044610 (2001).

3. M.A. Nagarajan, A.B. Balantekin, and N. Takigawa, Phys. Rev. C34, 894 (1986).

4. T. Rumin, K. Hagino, and N. Takigawa, Phys. Rev. C63, 044603 (2001).

5. K. Hagino and N. Rowley, Phys. Rev. C69, 054610 (2004).

6. Y. Aritomo, Phys. Rev. C 80, 064604 (2009).

7. V. Zagrebaev and W. Greiner, J. Phys. G 31, 825 (2005).

8. Y. Aritomo and M. Ohta, Nucl. Phys. A 744, 3 (2004).

9. K. Nishio et al., Phys,Rev. C 77, 064607 (2008): 82, 024611 (2010); 82, 044604 (2010).

10. Modified version of the CCFUL code, K. Hagino et al., Comput. Phys. Commun 123, 143 (1999).

11. Y. Aritomo, K. Hagino, K. Nishio and S. Chiba, Phys. Rev. C 85, 044614 (2012).

12. R. Bass, Nucl. Phys. A 231, 45 (1974); Nuclear Reactions with Heavy Ions (Springer, 1980).

13. R. du Rietz et al., Phys. Rev. Lett. 106, 052701 (2011).

14. A. Wakhle, Heavy Ion Accelerator Symposium 2012 Proceedings. 